\title{
Spherical harmonic analysis of particle velocity distribution function: Comparison of moments and anisotropies using Cluster data
}

\author{
Adolfo F. Viñas ${ }^{1}$ and Chris Gurgiolo ${ }^{2}$ \\ Received 22 July 2008; revised 22 July 2008; accepted 23 October 2008; published 22 January 2009.
}

[1] This paper presents a spherical harmonic analysis of the plasma velocity distribution function using high-angular, energy, and time resolution Cluster data obtained from the PEACE spectrometer instrument to demonstrate how this analysis models the particle distribution function and its moments and anisotropies. The results show that spherical harmonic analysis produced a robust physical representation model of the velocity distribution function, resolving the main features of the measured distributions. From the spherical harmonic analysis, a minimum set of nine spectral coefficients was obtained from which the moment (up to the heat flux), anisotropy, and asymmetry calculations of the velocity distribution function were obtained. The spherical harmonic method provides a potentially effective "compression" technique that can be easily carried out onboard a spacecraft to determine the moments and anisotropies of the particle velocity distribution function for any species. These calculations were implemented using three different approaches, namely, the standard traditional integration, the spherical harmonic (SPH) spectral coefficients integration, and the singular value decomposition (SVD) on the spherical harmonic methods. A comparison among the various methods shows that both SPH and SVD approaches provide remarkable agreement with the standard moment integration method.

Citation: Viñas, A. F., and C. Gurgiolo (2009), Spherical harmonic analysis of particle velocity distribution function: Comparison of moments and anisotropies using Cluster data, J. Geophys. Res., 114, A01105, doi:10.1029/2008JA013633.

\section{Introduction}

[2] The expansion of a plasma velocity distribution function as a sum of spherical harmonic functions provide a functional representation in terms of a set of linear spectral coefficients. These functions seem to form a natural representation since real measurements of the velocity distribution are taken in spherical coordinates. Furthermore, the Boltzmann equation that describes the evolution of the velocity distribution can be expressed as the well-known Sturm-Liouville form [Shkarofsky et al., 1966] which are suitable to a representation via orthogonal function solutions, such as the spherical harmonics. In a spherical harmonic representation, the set of spectral linear coefficients provide a complete description of the distribution fluid moments, anisotropies, and asymmetries. Spherical harmonics have been used in many problems of classical function analysis because they are both separable and orthogonal and they provide a useful representation of physical functions. Among other problems, these harmonic functions have been used in applications to describe the main internal magnetic field of the Earth [Langel, 1987] and other planets [Connerney, 1981; Connerney et al., 1991], as

\footnotetext{
${ }^{1}$ Heliophysics Science Division, Geospace Physics Laboratory, NASA Goddard Space Flight Center, Greenbelt, Maryland, USA.

${ }^{2}$ Bitterroot Basic Research, Hamilton, Montana, USA.

Copyright 2009 by the American Geophysical Union. 0148-0227/09/2008JA013633
}

well as to forward and inverse problems of solar differential rotation in helioseismology [Ritzwoller and Lavely, 1991; Christensen-Dalsgaardhen, 2002]. The level of description and accuracy of the spherical harmonic analysis model is mainly limited by the truncation of the series expansion. However, when expanding a velocity distribution for the purpose of obtaining the moments, the number of coefficients retained is determined by the highest moment to be resolved. As will be shown in the text, this is a small and manageable number.

[3] The spherical harmonic description of the velocity distribution function has been previously proposed as an approach to the solution of the Boltzmann equation (including the collision term) to provide a description of the angular integrated transport equations [Grad, 1949; Bayet et al., 1954; Jancel and Kahan, 1959a, 1959b, 1966; Johnson, 1960; Shakarofsky, 1963a, 1963b, 1963c; Shkarofsky et al., 1966]. Other expansions in terms of Hermite, Laguerre, and Sonine polynomials has also been suggested as solutions to the Boltzmann transport equation [Grad, 1949; Shakarofsky, 1963a; Jancel and Kahan, 1966; Grant and Feix, 1967a, 1967b]. However, applications of this approach using observed measurements to model the velocity distribution function, and to obtain an empirical determination of the moments of the distribution function have not been carried out before. This is due in part to the fact that earlier particle detectors lacked full $4 \pi$-steredian coverage and when they covered the full sphere often did not return a sufficient number of angular samples to allow for an accurate estimation of 
the expansion coefficients. Current particle spectrometers as those on the Cluster spacecrafts, and on future missions, such as MMS, have high enough angular resolution and sampling rate to allow the spherical harmonic expansion to be performed with an accuracy that should provide a robust determination of the moments, anisotropies, and asymmetries in the particle velocity distribution.

[4] Traditionally, modeling of the velocity distribution function has been done by a priori assumption of Maxwellian, kappa, Tsallis kappa-like or various combinations of these distribution function models which allows to determine some of the fluid moments quantities [Feldman et al., 1975; Pilipp et al., 1987; Maksimovic et al., 1997, 2005; Skoug et al., 2000; Nieves-Chinchilla and Viñas, 2008] from nonlinear fits to those distribution models. The spherical harmonic method provides a way to determine the full tensorial aspects of both the pressure tensor and even the higher-order heat flux, from coefficients which are always linear without requiring any iterative nonlinear scheme.

[5] The purpose of this work is to present a description of a method by which we can model the particle velocity distribution function and describe its kinetic structure, anisotropies, and moments from the spectral spherical harmonic coefficients using real satellite measurements. It also provide us with a reasonable and robust "compression" algorithm that can be carried out onboard any spacecraft for describing the structure, anisotropies, asymmetries, and moments of any particle velocity distribution function since only a small number of spectral coefficients needs to be determined and downloaded via telemetry. In this paper we follow the spherical harmonic approach and notation used by Bayet et al. [1954] and Jancel and Kahan [1966] throughout, which are the simplest and easiest to implement.

[6] The paper is organized as follows. Section 2 presents a brief description of the Cluster/PEACE electron spectrometer data measurements used in our study. Section 3 describes the standard numerical integration method implemented to compute the moments shown in this paper. Section 4 discusses the spherical harmonic theoretical model used to represent the velocity distribution function and its implementation to compute the moments from it. Section 5 shows a second approach to determine the spectral harmonic coefficients based upon the matrix method of singular value decomposition. Section 6 presents the application to actual data of the various methods and a comparison of the results. Section 7 presents an overall conclusions with a summary and discussion of our results.

\section{Cluster/PEACE Electron Velocity Distribution Data}

[7] The data used to test both the ability of the spherical harmonic series expansion to represent the velocity distribution and the accuracy of the moments derived from such expansion method compared to the standard numerical integration method were obtained from the PEACE Low Energy Electron Analyzer (LEEA) on-board Cluster-2 [Johnstone et al., 1997]. The time period selected was 2006/078 20:30:00 to 23:30:00 UT during which the spacecraft was upstream of the Earth's bow shock. During this period LEEA was returning a full three-dimensional electron distribution approximately every 4 seconds (satel- lite spin period) consisting of 30 energies $\times 12$ polar bins $\times$ 32 azimuthal sectors (11,520 samples). The energy range covered was from 7.3 to $3952 \mathrm{eV}$ in equal logarithmically spaced steps. The angular coverage was a full $4 \pi$ steredian ( $15^{\circ}$ polar and $11.25^{\circ}$ azimuthal bins).

[8] The moments calculations (both the standard and spherical harmonic based) in the paper are carries out at the full instrument temporal resolution of 4 seconds. Corrections for the spacecraft potential are made to the measured energies using the spin averaged potential supplied by the Electric Field and Wave (EFW) Experiment [Gustafsson et al., 1997]. A $-1 \mathrm{~V}$ contact potential is added to the measured potential. The total potential, which varies with the local plasma conditions, was between -3 and $-15 \mathrm{~V}$. Moments are computed in the local detector reference frame and then rotated into the GSE coordinate system. Higherorder moments (pressure, heat flux) are computed by shifting into the frame of the electron bulk velocity. When required, rotation into a magnetic field based coordinate system is made using the spin averaged magnetic field data supplied by the Fluxgate Magnetometer (FGM) experiment [Balogh et al., 1997].

\section{Standard Moment Computations}

[9] There are a number of methods available to numerically integrate a measured velocity distribution to estimate the plasma moments. What follows is a brief description of the method employed in the paper.

[10] Each data value which makes up the measured velocity distribution has associated with it a velocity range $\left(v_{1}\right.$ to $\left.v_{2}\right)$, an elevation range $\left(\theta_{1}\right.$ to $\left.\theta_{2}\right)$ and an azimuthal angle $(\phi)$. We make two assumptions. First, within a constant velocity shell the phase space density varies linearly between successive azimuths as

$$
f=A \phi+B
$$

Second, the phase space density has no variation in either velocity or elevation within the ranges covered by the measurement. Under these assumptions all of the moments can be reduced to a sum of exact integrals over the phase space volumes occupied by each measurement.

[11] As an example consider the plasma density. The general formula is

$$
n=\int_{0}^{\infty} \int_{0}^{2 \pi} \int_{0}^{\pi} f(v, \theta, \phi) v^{2} \sin (\theta) d v d \phi d \theta
$$

which can be rewritten as

$$
n=\sum_{i=0}^{i=l} \int_{v_{i a}}^{v_{i b}} \int_{\theta_{i a}}^{\theta_{i b}} \int_{\phi_{i a}}^{\phi_{i b}}\left(A_{i} \phi+B_{i}\right) v^{2} \sin (\theta) d v d \phi d \theta
$$

where the sum is over all of the points making up the distribution function. Performing the integrals, this reduces to:

$$
n=\frac{1}{3} \sum_{i=0}^{i=l}\left(v_{i b}^{3}-v_{i a}^{3}\right)\left(\cos \theta_{i a}-\cos \theta_{i b}\right)\left(\frac{A_{i}}{2}\left(\phi_{i b}^{2}-\phi_{i a}^{2}\right)+B_{i}\left(\phi_{i b}-\phi_{i a}\right)\right)
$$


which can be solved directly knowing the ranges covered by each element in the distribution as well as the A and B coefficients. Higher-order moments are solved for in exactly the same manner. These are not shown but the approach is similar and should be obvious.

\section{Spherical Harmonic Method}

[12] In this section we describe the spherical harmonic expansion method used to model the particle velocity distribution function $f_{s}(v, \theta, \phi)$, where the subscript $\mathrm{s}$ represents the particle specie, $\mathrm{v}$ is the particle velocity and the variables $(\theta, \phi)$ are the elevation and azimuth angles, respectively. The polar and azimuthal coordinates $(\theta, \phi)$ are measured in a right-handed coordinate system with $\phi$ measured from the positive $x$ axis and $\theta$ measure from the positive $z$ axis. We will also show how the spectral coefficients in the expansion are directly related to the moments of the velocity distribution.

[13] The general spherical harmonic representation of the particle velocity distribution (following the notation and approach by Bayet et al. [1954] and Jancel and Kahan [1966]) is given by

$$
f_{s}(v, \theta, \phi)=a_{0,0}(v)+\sum_{l=1}^{l=\infty} \sum_{m=0}^{m=l}\left[a_{l, m}(v) C_{l, m}+b_{l, m}(v) S_{l, m}\right]
$$

where $C_{l, m}$ and $S_{l, m}$ are spherical harmonic functions defined as

$C_{l, m}(v, \theta, \phi)=v^{l} P_{l}^{m}(\cos \theta) \cos (m \phi)$

$S_{l, m}(v, \theta, \phi)=v^{l} P_{l}^{m}(\cos \theta) \sin (m \phi)$

The spectral spherical harmonic coefficients $a_{0,0}, a_{l, m}$, and $b_{l, m}$ depend only on the magnitude of $\mathrm{v}$. The functions $P_{l}^{m}(\cos \theta)$ are the associated Legendre polynomials of order $(l, m)$. In general, the summation over $l$ runs from $l=0$ to $\infty$, but in this application its value is bounded $\left(l=l_{\max }\right)$ by the highest moment of interest.

[14] Since we have chosen to represent the expansion by two set of coefficients $a_{l, m}$ and $b_{l, m}$ the summation over $m$ runs only from 0 to $l$. Higher $l$ and $m$ values represent more complex anisotropies and moments in the velocity distribution function. The number of coefficients produced in the expansion are

$$
N=1+\sum_{l=1}^{l=l_{\max }}(2 l+1)
$$

To represent moments up through the pressure tensor (which is $l_{\max }=2$ ) requires 9 coefficients. The same number of coefficients will also allow for the representation of the heat flux vector. One advantage of using spherical harmonics is that they are orthogonal over the whole velocity space of radius v. In other words, the integral of $C_{l, m} S_{l, m^{\prime}}$ over such a sphere is always zero, as are all integrals of the products $C_{l, m} C_{l^{\prime}, m^{\prime}}$ and $S_{l, m} S_{l^{\prime}, m^{\prime}}$, except when $l=l^{\prime}$ and $m=m^{\prime}$. Defining the surface element $d S=v^{2} d \Omega$ in the later case, the resulting normalization conditions are

$$
\int\left(C_{l, m}\right)^{2} d S=\int\left(S_{l, m}\right)^{2} d S=v^{2(l+1)} \frac{2 \pi\left(1+\delta_{m, 0}\right)(l+m) !}{(2 l+1)(l-m) !}
$$

and

$$
\int\left(C_{1,0}\right)^{2} d S=v^{2(l+1)} \frac{4 \pi}{(2 l+1)}
$$

for $m=0$. On the basis of the orthogonality condition of the basis functions $C_{l, m}$ and $S_{l, m}$ the spectral coefficients $a_{l, m}$ and $b_{l, m}$ are determined by

$$
\begin{aligned}
a_{l, m}(v)= & \frac{(2 l+1)(l-m) !}{2 \pi\left(1+\delta_{m, 0}\right)(l+m) ! v^{l}} \\
& \cdot \iint f(v, \theta, \phi) P_{l}^{m}(\cos \theta) \cos (m \phi) d \Omega \\
b_{l, m}(v)= & \frac{(2 l+1)(l-m) !}{2 \pi\left(1+\delta_{m, 0}\right)(l+m) ! v^{l}} \\
& \cdot \iint f(v, \theta, \phi) P_{l}^{m}(\cos \theta) \sin (m \phi) d \Omega
\end{aligned}
$$

where $\delta_{m, 0}$ is the Kronecker delta and the integration is carried out over the solid angle $d \Omega=\sin \theta d \theta d \phi$ for $0 \leq \theta \leq$ $\pi$ and $0 \leq \phi \leq 2 \pi$.

[15] How closely the spherical harmonic expansion describes the distribution function depends on two factors: the number of coefficients $a_{l, m}$ and $b_{l, m}$ used in the fit and the number of angular samples in the data at a fixed velocity. The larger the number of coefficients used the better the fit and the larger number of angular samples available the better resolved will be the coefficients. As will become evident below, however, if you just want to resolve the macroscopic moments of the distribution function without concerns about its fine scale structure all that is needed are 9 spectral coefficients at each energy channel.

[16] To see how this works consider the moment density $n_{s}$. This is determined from the integral

$$
n_{s}=\iint f_{s}(v, \theta, \phi) v^{2} d v d \Omega
$$

which can be shown to be related only to the $a_{0,0}$ coefficient (the isotropic term). Substituting the series expansion in equations (4) and (5) and using the orthogonal properties given in equations (7), (8) and (9) we obtain

$$
n_{s}=\int_{0}^{\infty} a_{0,0} d^{3} v=4 \pi \int_{0}^{\infty} a_{0,0} v^{2} d v
$$

The same is also true when calculating the mean kinetic energy density $K_{s}=n_{s}\langle E\rangle$ as:

$$
K_{s}=n_{s}\langle E\rangle=\int_{0}^{\infty} \frac{1}{2} m v^{2} a_{0,0} 4 \pi v^{2} d v
$$


Similar steps can be invoked to determine the components of the average velocity which is given by the equation

$$
\boldsymbol{U}_{s}=\frac{1}{n_{s}} \iint f_{s}(v, \theta, \phi) \mathbf{v} v^{2} d v d \Omega
$$

The velocity vector $\mathbf{v}$ can be expressed as a function of firstorder spherical functions (i.e., first-order anisotropies) as follows:

$$
\mathbf{v}=C_{1,1} \hat{e_{x}}+S_{1,1} \hat{e_{y}}+C_{1,0} \hat{e_{z}}
$$

where $\hat{\mathbf{e}}=\left(\hat{e_{x}}, \hat{e_{y}}, \hat{e_{z}}\right)$ is a Cartesian unit vector. Combining expressions (4), (5), and (14) with equation (13) and after some analytical algebraic calculations using equations (7), (8), and (9) gives

$$
\mathbf{U}_{s}=\frac{4 \pi}{3 n_{s}} \int_{0}^{\infty}\left(\begin{array}{l}
a_{1,1} \\
b_{1,1} \\
a_{1,0}
\end{array}\right) v^{4} d v
$$

where the quantity between the parenthesis denotes the component of a vector in the coordinate system of $\hat{\mathbf{e}}$ and shows that only the $l=1$ terms contribute to the moment.

[17] The pressure tensor moment is given by:

$$
\mathbf{P}_{s}=m_{s} \iint f_{s}(v, \theta, \phi)\left(\mathbf{v}-\mathbf{U}_{s}\right)\left(\mathbf{v}-\mathbf{U}_{s}\right) v^{2} d v d \Omega
$$

Substituting equations (4), (5), and (14) and using equations (7), (8), and (9) into equation (15) we can express equation (15) as the sum of three terms which represent the $l=0,1$, and 2 contributions. The $l=0$ (zeroth-order isotropic) component is given by:

$$
\Psi_{s}^{(0)}=\frac{2}{3} n_{s}\langle E\rangle \mathbf{I}
$$

the $l=1$ (first-order anisotropies) component by:

$$
\Psi_{s}^{(1)}=-n_{s} m_{s} \mathbf{U}_{s} \mathbf{U}_{s}
$$

and after some algebraic manipulation, the $l=2$ (secondorder anisotropies) component by

$$
\Psi_{s}^{(2)}=\frac{4 \pi}{15} m_{s} \int_{0}^{\infty}\left(\begin{array}{ccc}
-a_{2,0}+6 a_{2,2} & 6 b_{2,2} & 3 a_{2,1} \\
6 b_{2,2} & -a_{2,0}-6 a_{2,2} & 3 b_{2,1} \\
3 a_{2,1} & 3 b_{2,1} & 2 a_{2,0}
\end{array}\right) v^{6} d v
$$

The full pressure tensor is expressed as

$$
\begin{aligned}
\mathbf{P}_{s}= & \frac{2}{3} n_{s}\langle E\rangle \mathbf{I}-n_{s} m_{s} \mathbf{U}_{s} \mathbf{U}_{s}+\frac{4 \pi}{15} m_{s} \\
& \cdot \int_{0}^{\infty}\left(\begin{array}{ccc}
-a_{2,0}+6 a_{2,2} & 6 b_{2,2} & 3 a_{2,1} \\
6 b_{2,2} & -a_{2,0}-6 a_{2,2} & 3 b_{2,1} \\
3 a_{2,1} & 3 b_{2,1} & 2 a_{2,0}
\end{array}\right) v^{6} d v
\end{aligned}
$$

If the plasma is isotropic only the $\boldsymbol{\Psi}_{s}^{(0)}$ term contributes to the pressure, otherwise there are contributions from all three terms. When the pressure contains a local symmetry of revolution the tensor can be diagonalized.

[18] The highest moment considered in this paper is the heat flux which is given by:

$$
\mathbf{Q}_{s}=m_{s} \iint\left(\mathbf{v}-\mathbf{U}_{s}\right)\left(\mathbf{v}-\mathbf{U}_{s}\right)\left(\mathbf{v}-\mathbf{U}_{s}\right) f_{s}(v, \theta, \phi) v^{2} d v d \Omega
$$

The heat flux is a third-order tensor. It is more convenient to consider it in vector form which partially describes the anisotropies and asymmetries in the thermal flux of the particles. Contracting over two indices reduces the tensor $\mathbf{Q}_{s}$ to a heat flux vector and introduces a factor of $1 / 2$ as follows:

$$
\begin{aligned}
\mathbf{q}_{s}= & \frac{m_{s}}{2} \iint\left(\mathbf{v}-\mathbf{U}_{s}\right)\left(v-U_{s}\right)^{2} f_{s}(v, \theta, \phi) v^{2} d v d \Omega \\
= & \frac{m_{s}}{2} \iint\left(\mathbf{v} v^{2} f_{s}(v, \theta, \phi) v^{2} d v d \Omega-\left(\frac{1}{2} n_{s} m_{s} U_{s}^{2}\right) \mathbf{U}_{s}\right. \\
& -\left(\frac{1}{2}\left(\operatorname{Tr} \boldsymbol{P}_{s}\right) \mathbf{I}+\mathbf{P}_{s}\right) \cdot \mathbf{U}_{s}
\end{aligned}
$$

Substituting equations (4), (5), and (14) into equation (21) and making use of the conditions in equations (7), (8), and (9) the resultant spherical harmonic reduced heat flux vector becomes

$$
\begin{aligned}
\mathbf{q}_{s}= & \frac{m_{s}}{2}\left(\frac{4 \pi}{3}\right) \int_{0}^{\infty}\left(\begin{array}{l}
a_{1,1} \\
b_{1,1} \\
a_{1,0}
\end{array}\right) v^{6} d v-\left(\frac{1}{2} n_{s} m_{s} U_{s}^{2}\right) \mathbf{U}_{s} \\
& -\left(\frac{1}{2}\left(\operatorname{Tr} \boldsymbol{P}_{s}\right) \mathbf{I}+\mathbf{P}_{s}\right) \cdot \mathbf{U}_{s}
\end{aligned}
$$

where $\operatorname{Tr} \mathbf{P}_{s}$ is the trace of the pressure tensor. While the full heat flux tensor corresponds to the inclusion of the $1=3$ coefficients in the expansion, reducing it to a vector quantity (by contraction of the tensor $\mathbf{Q}_{\mathbf{s}}$ ) gives a result that only depends on the first-order anisotropies plus the contribution from the isotropic part. The second and third-order anisotropies vanish exactly.

\section{Spherical Harmonic Analysis and Singular Value Decomposition}

[19] The previous section showed that the derivation of the plasma moments from the spherical harmonic expansion reduces to the determination of the $a_{0,0}, a_{l, m}$, and $b_{l, m}$ linear spectral coefficients. These spectral coefficients can be obtained via two methods: (1) by quadrature of the spherical harmonics coefficients as presented in the previous section in equation (9) and (2) by posing the determination of the coefficients as a matrix problem through a system of coupled equations directly obtained from equation (4) above, and then solving the system given by

$$
\mathbf{M} \cdot \mathbf{c}=\mathbf{r}
$$

where $\mathrm{c}$ is a vector containing the unknown spectral coefficients $a_{0,0}, a_{l, m}$, and $b_{l, m}$ up to $1=2$, the matrix $\mathbf{M}$ is formed by the spherical harmonic functions $C_{l, m}$ and $S_{l, m}$, and the vector $\mathbf{r}$ is formed by the measured values of the 


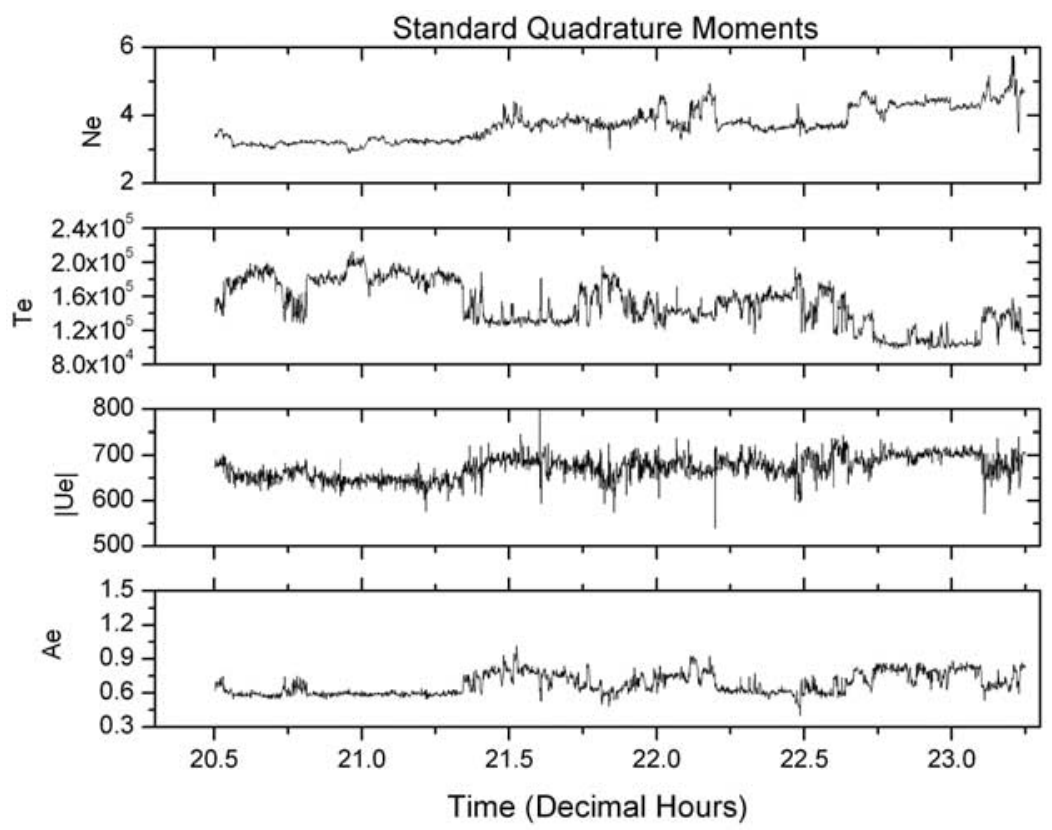

Figure 1. Overview of time period showing moments computed using the standard numerical integration technique.

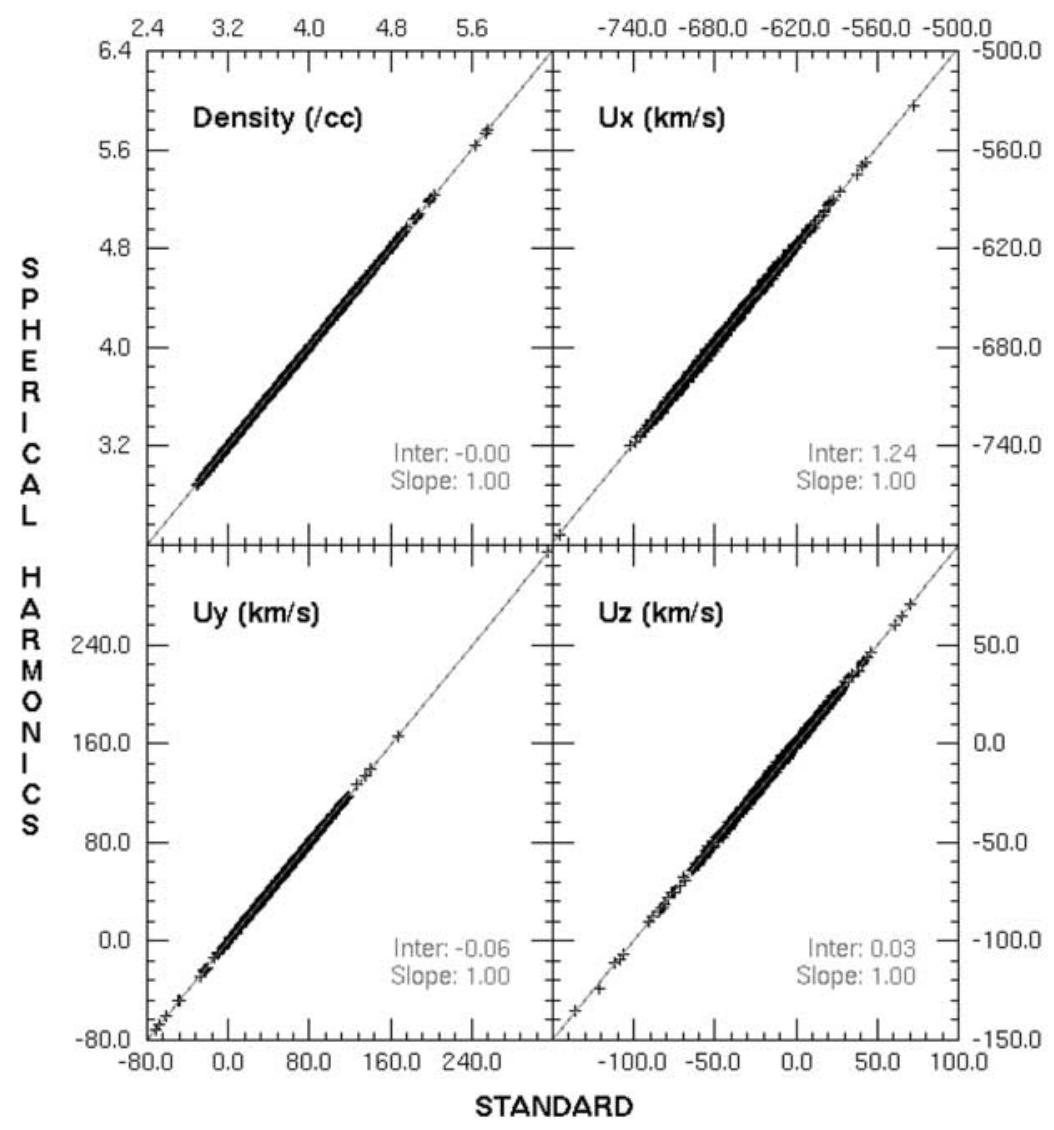

Figure 2. Comparisons of the density and bulk velocity moments computed using the spherical harmonic method and the standard numerical integration method. $x$ axis labels for the top plots are along the top, and $y$ axis labels for the right-hand plots are along the right $y$ axis. 


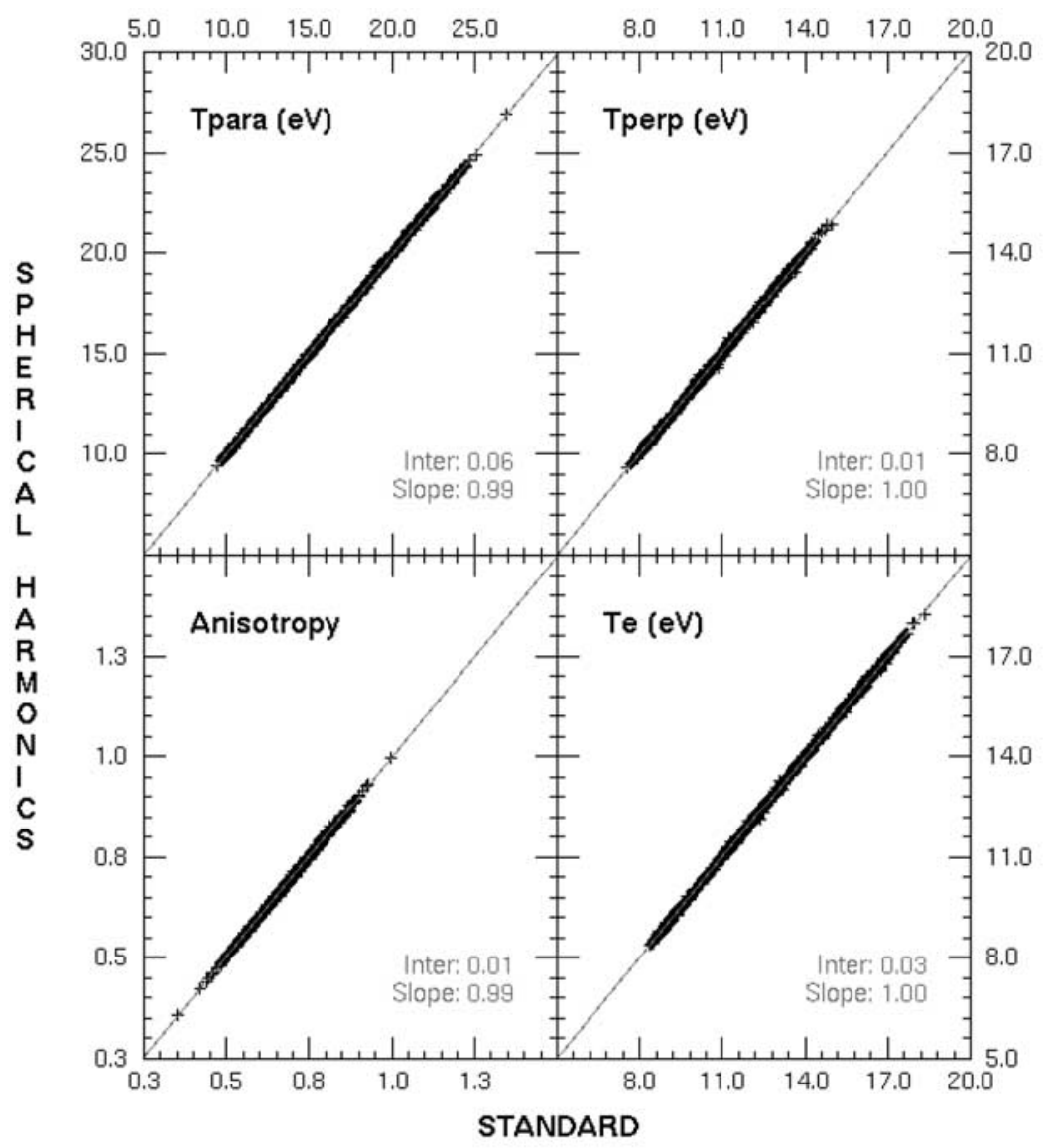

Figure 3. Comparisons of aspects of the plasma temperature computed using the spherical harmonic method and the standard numerical integration method. $x$ axis labels for the top plots are along the top, and $y$ axis labels for the right-hand plots are along the right $y$ axis.

velocity distribution function $f_{s}(v, \theta, \varphi)$. There are instances where the matrix system of equation (23) contains less (nonzero) data values than the number of spectral coefficients at a constant speed shell. For these cases, the number of spectral coefficients being solved for are set to zero in the analysis since we are only interested on posing the matrix problem as an overdetermined system. Therefore forming the inner product of the spherical harmonic functions and the measured values of the velocity distribution $f_{s}$ the solution of the matrix system above is obtained as follows

$$
\mathbf{c}=\left(\mathbf{M}^{T} \cdot \mathbf{M}\right)^{-1} \mathbf{M}^{T} \cdot \mathbf{r}
$$

[20] The matrix $\mathbf{M}$ is not necessarily a square matrix and its inverse may be difficult to estimate since some of its eigenvalues may be zero or close to machine zero. Thus since the spectral coefficients are linear and independent, we solved the system of equations in equation (23) via the singular value decomposition (SVD) method [Press et al., 1999] which is more immune method to numerical errors.

[21] SVD exposes the geometric structure of a matrix, an important aspect of many matrix calculations. A matrix can be described as a transformation from one vector space (e.g., vector $\mathbf{M} \cdot \mathbf{c}$ ) to another (e.g., vector $\mathbf{r})$. The components of the SVD quantify the resulting change between the underlying geometry of those vector spaces. The singular value decomposition of a matrix $\mathbf{M}$ expresses $\mathbf{M}$ as a product of three matrices, i.e., $\mathbf{M}=\mathbf{U} \boldsymbol{\Lambda} \mathbf{V}^{T}$ in such a way that the matrices $\mathbf{U}$ and $\mathbf{V}$ are orthogonal in the sense that their column vectors are orthonormal (i.e., $\mathbf{U}^{T} \mathbf{U}=\mathbf{I}$ and $\mathbf{V}^{T} \mathbf{V}=\mathbf{I}$ ). The matrix $\boldsymbol{\Lambda}$ is a diagonal matrix formed by the singular values or eigenvalues of the system $\mathbf{M}^{T} \mathbf{M}$ that may or may not be zero (in the numerical sense). If the matrix $\mathbf{M}$ is singular or close to it in the numerical sense (i.e., has any of its eigenvalues equal to zero or machine zero) then there is some subspace of $\mathrm{c}$ that is mapped to zero, called a null space. This subspace needs to be removed in order to obtain an optimal solution to the problem as in equation (24) above. The details of the SVD method will not be further discussed here however, in summary obtaining these singular values (if any) and removing their effects in the projection from one vector space to the other is what entails the robust determination of the spectral coefficient. Thus to determine the moments up to the pressure tensor, 9 independent spectral coefficients at each constant speed shell are required and such SVD matrix analysis needs to be carried out for each energy forming an overdetermined system at all instances.

\section{Cluster/PEACE Moment Comparison Results}

[22] Figure 1 shows an overview of the time period used in the analysis. Figure 1 is a set of 4 time plots, showing 


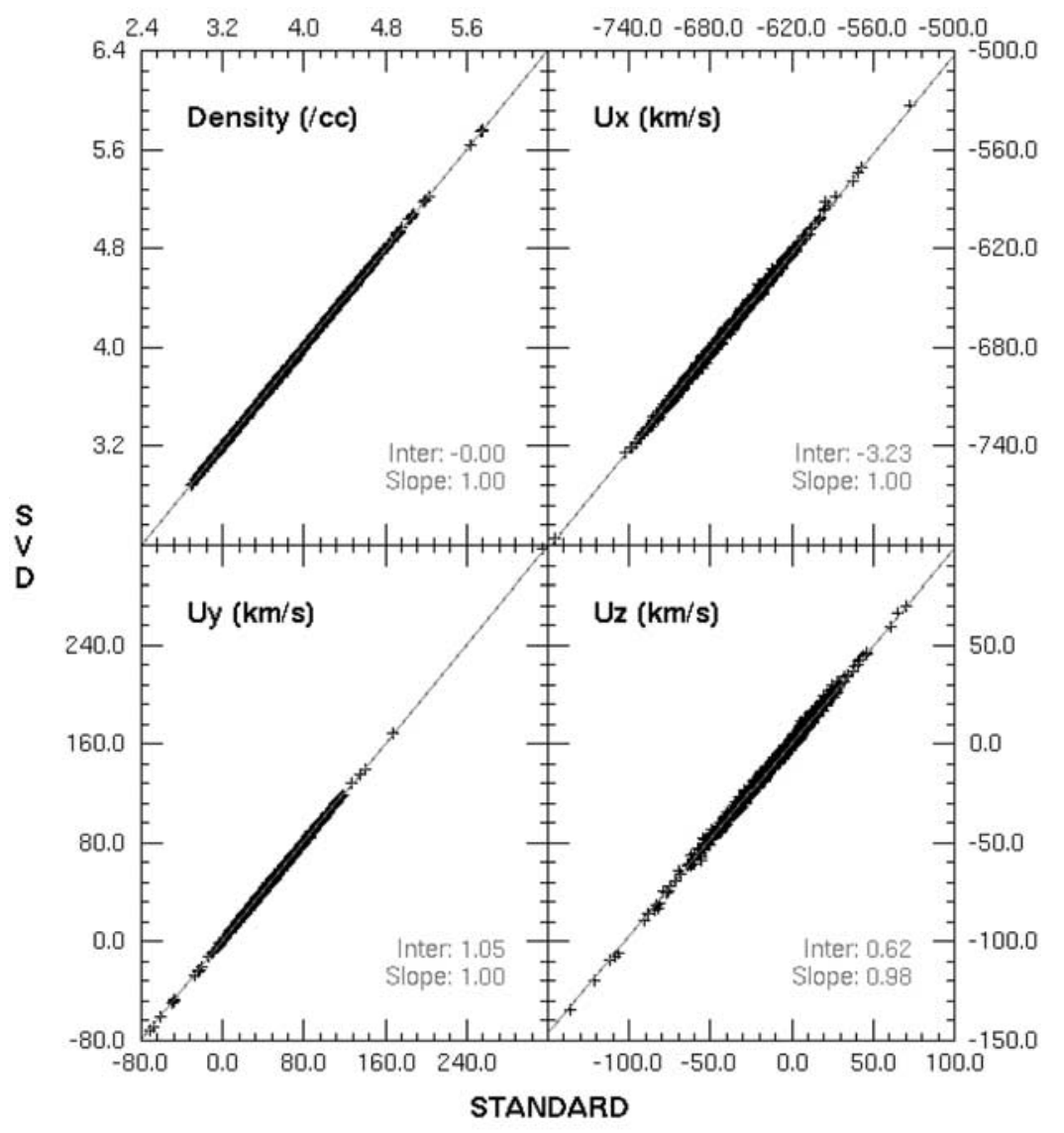

Figure 4. Comparisons of the density and bulk velocity moments computed using the singular value decomposition spherical harmonic method and the standard numerical integration method. $x$ axis labels for the top plots are along the top, and $y$ axis labels for the right-hand plots are along the right $y$ axis.

from top to bottom, the electron plasma moment density $\left(\mathrm{N}_{e}\right)$, the plasma temperature $\left(\mathrm{T}_{e}\right)$, bulk velocity magnitude $\left(\mathrm{U}_{e}\right)$, and temperature anisotropy $\left(A_{e}=T_{\perp} / T_{\|}\right)$. The moments in Figure 1 were computed using the traditional numerical integration method described above in section 3 . Our results show that essentially, the same set of moments were derived using the spherical harmonic method.

[23] The time period was selected because Cluster-2 was returning data in burst mode and PEACE was returning a three-dimensional distribution once per spin with 384 angular samples per energy step. Any similar set of data would have served our purpose equally well. Each distribution function measured within the time period (about 2700 data measurements) was expanded as a series of spherical harmonics and the spectral coefficients $a_{l, m}$ and $b_{l, m}$ (defined in equation (9) above) computed out to order $l=2$. The computations were made using a similar numerical integration procedure as described in section 3 .

[24] How well the spherical harmonic method is able to reproduce the plasma moments is demonstrated in Figures 2 and 3. Figure 2 shows a comparison between the moment density $\left(\mathrm{N}_{e}\right)$ and bulk velocity components $\left(\mathrm{U}_{x}, \mathrm{U}_{y}, \mathrm{U}_{z}\right)$ estimated by both the standard numerical integration method and by the spherical harmonic procedure. The data in each plot have been fit to a first-order polynomial which is shown as a red line. The slope and intercept of the fit is listed in each plot box. It is evident from the plots that the moments estimated using the spherical harmonic method match those computed through the standard numerical integration to a very high degree of accuracy. The linear relation between these physical quantities indicate the high degree of correlation and the small dispersion is indicative of small errors and the accuracy of the moment estimates. This is also indicated by the slope of the linear fit and the small dispersion of the data about the fit.

[25] Figure 3 has a similar format as Figure 2 but contains plots of aspects of the plasma temperature which test the accuracy of the estimates of the pressure tensor. Clockwise from the top left plot are the parallel and perpendicular plasma temperatures in the magnetic field coordinate system, the total temperature, and the temperature anisotropy defined as $T_{\perp} / T_{\|}$. The temperatures are computed from $\mathrm{P}$ (i.e., the plasma pressure) using $T=P / N_{e} k_{B}$. The perpendicular and parallel temperatures are formed as

$$
T_{\|}=(\vec{b} \cdot \mathbf{P} \cdot \vec{b}) /\left(N_{e} k_{B}\right), \quad T_{\perp}=\frac{1}{2}\left(\operatorname{Tr} \mathbf{P}-P_{\|}\right) /\left(N_{e} k_{B}\right)
$$

where $\vec{b}$ is the magnetic field unit vector, $\mathrm{k}_{B}$ is the Boltzmann constant, and $\operatorname{Tr} \mathbf{P}$ is the trace of the pressure tensor. The anisotropy is given by $A_{e}=T_{\perp} / T_{\|}$. As with the density and velocity moments, the plasma temperature (which is essentially a proxy for the pressure tensor) shows a very high correlation between the two computational 


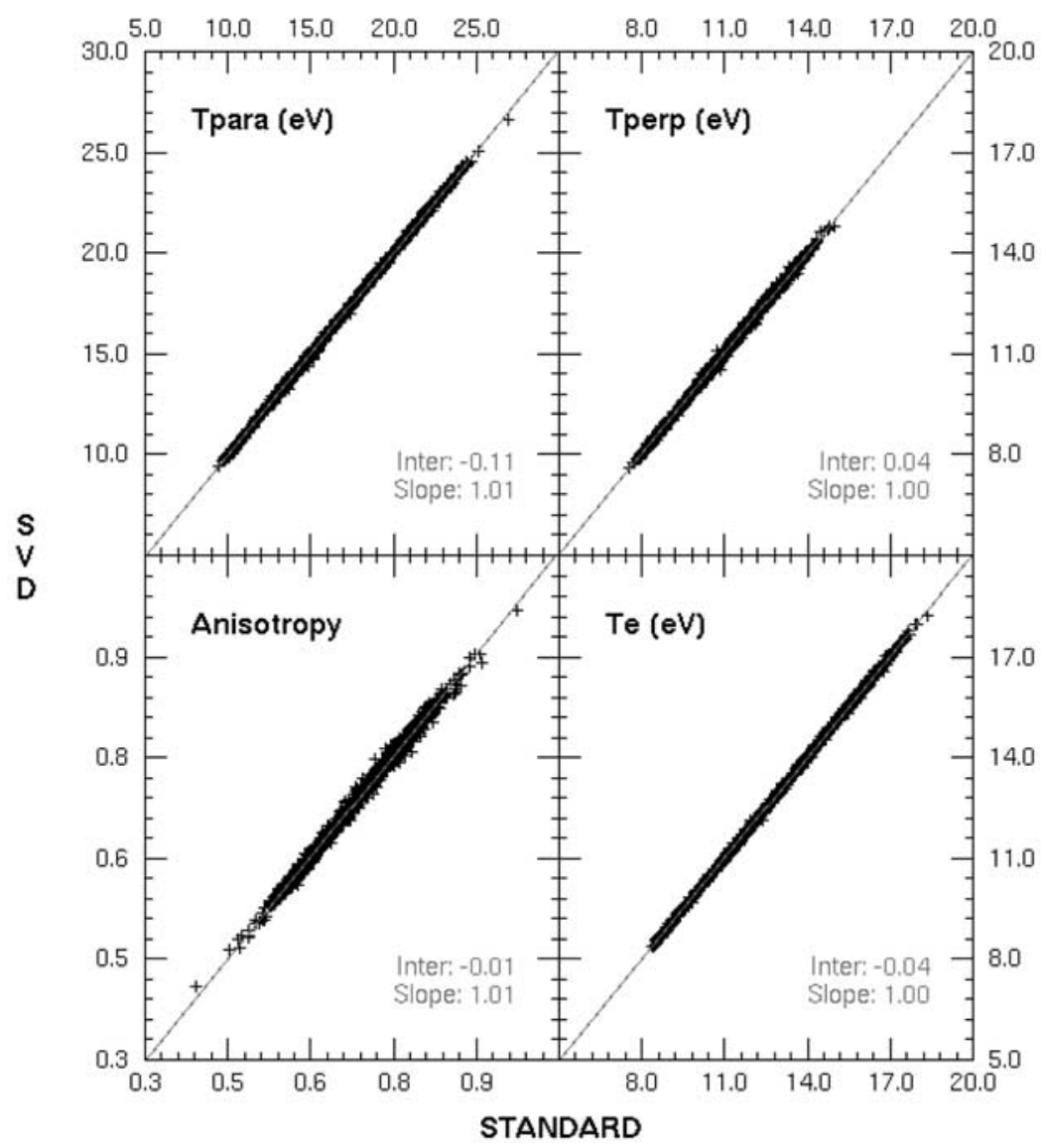

Figure 5. Comparisons of aspects of the plasma temperature computed using the singular value decomposition spherical harmonic method and the standard numerical integration method. $x$ axis labels for the top plots are along the top, and $y$ axis labels for the right-hand plots are along the right $y$ axis.

methods. Plots of the individual components of the pressure tensor which are not included in the paper show the same high correlation. The results shown above in Figures 2 and 3 are clear evidence that computation of plasma moments by spherical harmonics, at least during the time selected, is equivalent to computation by the standard method or numerical integration.

[26] Figures 4 and 5 are similar to Figures 2 and 3 but show the results of the moments and anisotropy calculations computed from the expansion coefficients using the singular value decomposition procedure versus the moments estimated by the standard method. It appears at first glance, that because the spectral coefficients are coupled (i.e., are not fully independent) by means of a system of linear equations (SVD), it may produce significantly more scatter in the moment solutions since the statistical variations in the data could be strongly reflected back into the solutions for the expansion coefficients. However, as we see in Figures 4 and 5 , this is misleading since the solution obtained is as accurate and robust as those determined by the SPH method in Figures 2 and 3. Figures 4 and 5 show some scatter (less than $2 \%$ ) in the moment and anisotropies showing results that are remarkably similar to their analog in the SPH method. The reason for this is that the SVD procedure more closely resembles the orthogonal properties of the spherical harmonic method by obtaining a solution vector (c), without requiring any integration, that minimizes the residual vector $|\mathrm{M} \cdot \mathrm{c}-\mathrm{r}|$ via a decomposition of the matrix system $\mathrm{M}$ into orthogonal matrix systems $\mathrm{U}$ and $\mathrm{V}$ as discussed in section 5. This residual minimization is almost analogous to the standard minima results obtained via the least squares method. In comparison, when solving for the coefficients in equation (9), these spectral coefficients enter fully independent of each other and their integration in equation (9) tends to smooth or average out small statistical variations in the data. In this sense, the procedure resembles more closely the standard traditional method used to derive the moments themselves via numerical integration.

[27] How well the spherical harmonic expansion fits the measured distribution is illustrated in Figures 6 and 7 . Figure 6 shows an angular overview of a measured velocity distribution at 8 (of the possible 30) energy shells. Each plot is a "skymap" of the velocity distribution in the instrument frame of reference with azimuth $(\phi)$ plotted along $\mathrm{X}$ and elevation $(\theta)$ plotted along Y. The Sun is located in the plane defined by $\phi=0$. The largest energy shell shown is in the top left plot and the lowest in the bottom right plot. Annotation to the right of each plot shows the center energy of the shell and the maximum and minimum values in the plot. The color scale for each plot runs from the minimum to the maximum values of the distribution function, thus you cannot directly compare the intensities between plots simply 


\section{Cluster Skymap of Electron Velocity Distribution Function (DATA)}

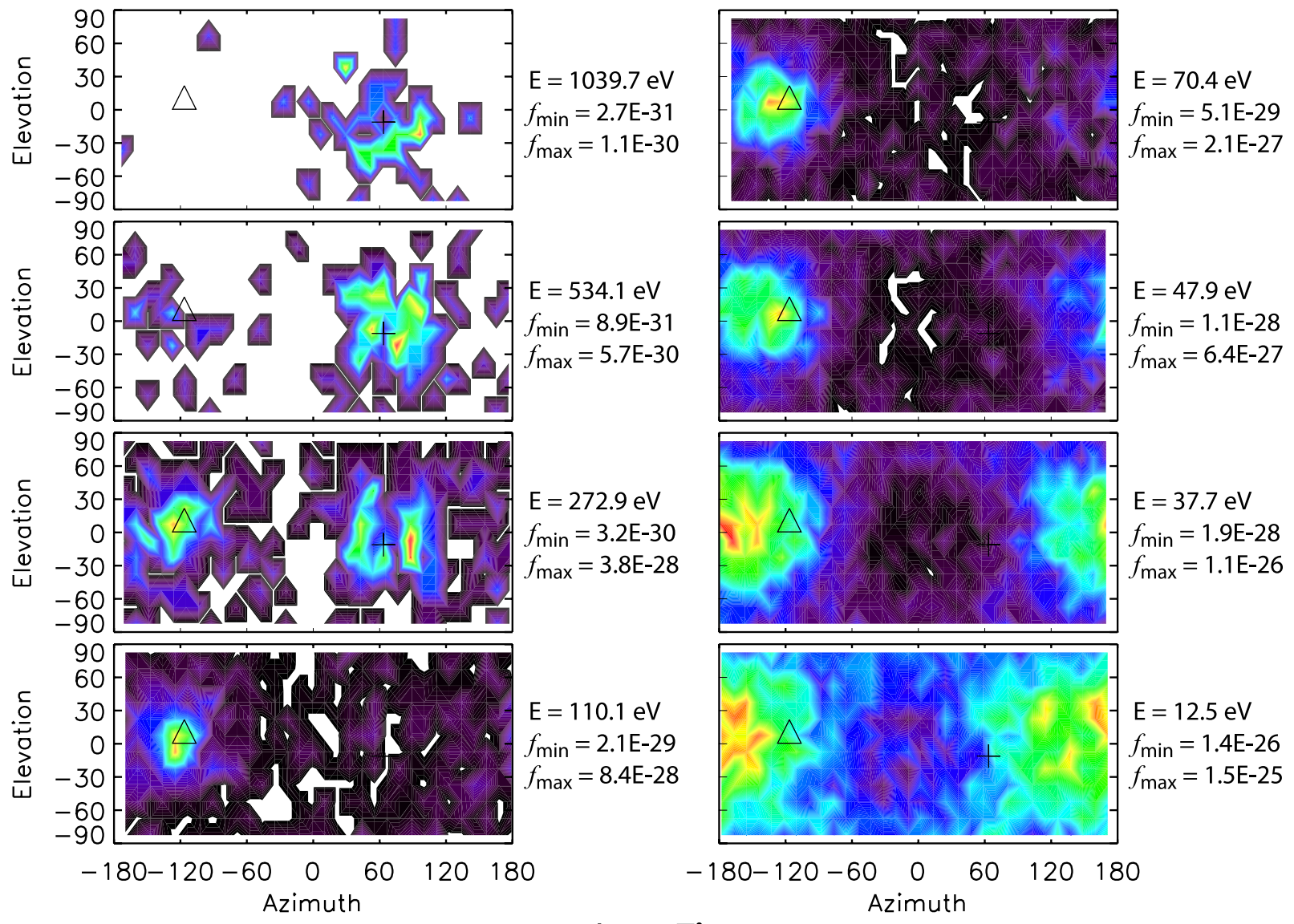

\section{6/078 Time 20:45:08}

Figure 6. Skymap multipanel plots of Cluster/PEACE data measurements of the velocity distribution function as a function of azimuth and elevation for different energy channels. Data are represented in a frame where the sun is at $(0,0)$.

by color. The plus $(+)$ and triangle symbols in each plot are the projection of the head and tail of the spin averaged magnetic field vector respectively. Figure 6 shows the fine details present in the velocity distribution as a function of the angular sampling of the particle detector. The lowest two energies plotted (12.5 to $37.7 \mathrm{eV}$ ) shows a typical solar wind core-halo distribution. As the strahl begins to emerge and dominate the distribution in the next two energies the distribution begins to shift toward being field aligned and by $110.1 \mathrm{eV}$ the strahl is the only population present. At $272.9 \mathrm{eV}$ a second population can be seen flowing opposite to the strahl. These are reflected electron, presumably off the shock. Figure 7 displays a spherical harmonic model representation of the same data measurements as shown in Figure 6. The model has smoothed the measured distribution but retains its main features. The core, halo, strahl, and reflected populations can be easily identified and the model has similar angular widths as seen in the data. The smoothing appears to have only an extremely minimal effect on the determination of the moments. This may be due to the fact that the portions of the distributions which significantly contribute to the moments are themselves reasonably smooth and well defined. Thus it is remarkable that the spherical harmonic representation of the velocity distribu- tion picks up the main details of the measured distributions. Obviously, expanding the series to include more coefficients would provide a model which showed more of the fine details in the measured distribution, but this would not have any effect on the derived moments which use only the first 9 coefficients.

[28] Figure 8 shows the same distribution as in Figure 6 but in this case we have run the spherical harmonic expansion out to $l_{\max }=5$ (which uses 36 coefficients per energy channel). There are now more smoothed fine structures seen in the velocity distribution function. This is very evident in the $1040 \mathrm{eV}$ channel.

\section{Summary and Discussion}

[29] Observations of the electron velocity distribution functions upstream of the Earth's bow shock from the PEACE particle spectrometer aboard Cluster have provided an opportunity to model the velocity distribution function and to examine a new method to determine the electron moments, asymmetries and anisotropies.

[30] Analysis shows that the electron moments and anisotropies calculated by both the quadrature of the spectral coefficients of the spherical harmonic method and by 


\section{Cluster Skymap of Electron Velocity Distribution Function}

(SPH MODEL Lmax=2)
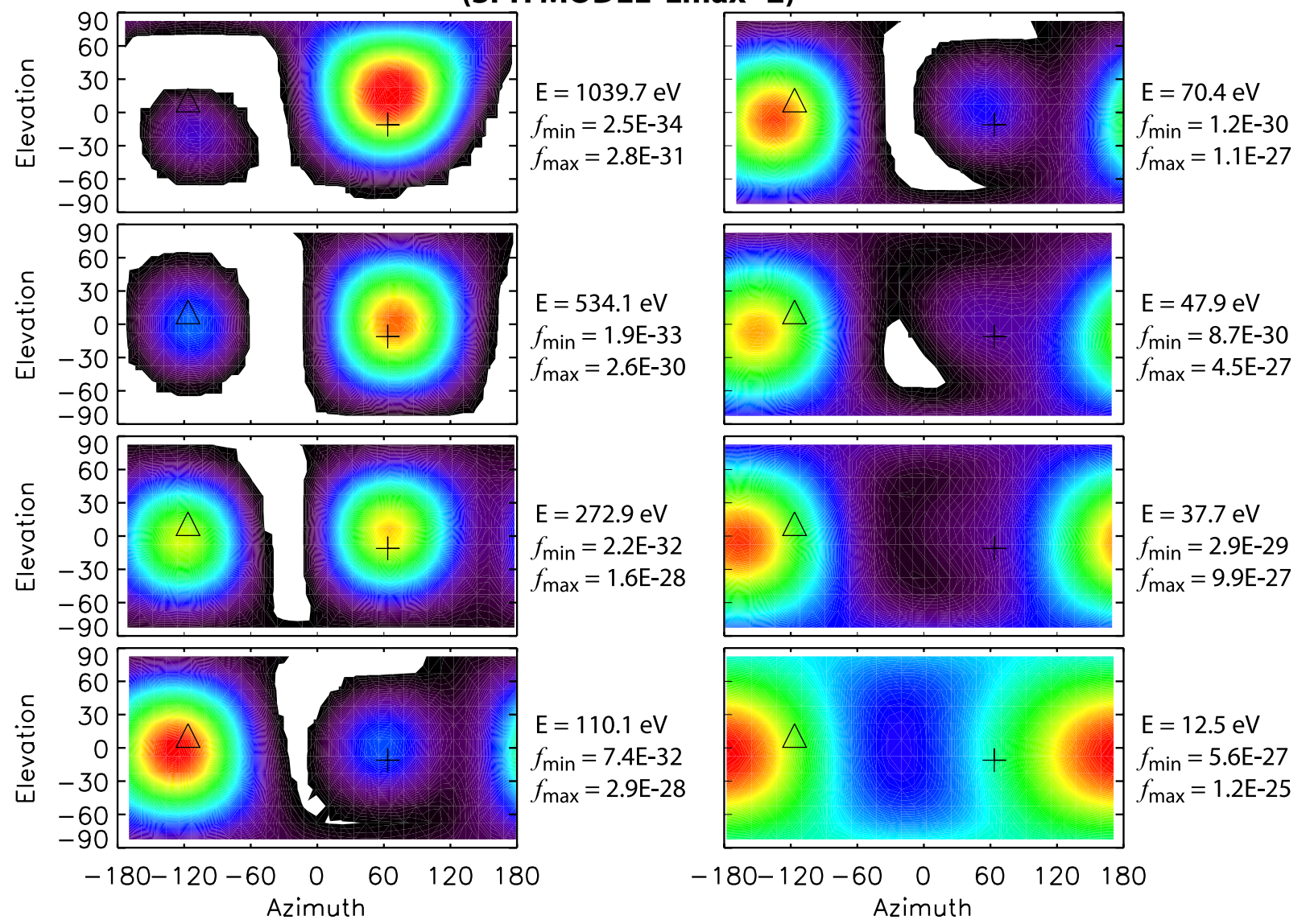

$-180-120-60 \quad 0 \quad 60 \quad 120 \quad 180$

\section{6/078 Time 20:45:08}

Figure 7. Skymap multipanel plots of the spherical harmonic model for $l_{\max }=2$ (9 coefficients) of the velocity distribution function as a function of azimuth and elevation for different energy channels. Data are represented in the same frame as in Figure 6.

singular value decomposition are in remarkable agreement with the standard moment integration method as indicated in Figures 2-5. The overall trend of the moments matched those computed in the standard method with some acceptable insignificant spread about the trend for both the SPH and SVD methods.

[31] The spherical harmonic analysis also provided a reasonable and simple physical representation of the velocity distribution function itself without the need to impose an a priori model representation of the distribution based upon Maxwellians, kappa or Tsallis kappa-like distribution function models. The method only requires the determination of nine spectral coefficients for each speed shell to have a smooth resolved velocity distribution function model. More spectral coefficients are required if you want the full heat flux tensor (and not just the trace) or if you want to resolve fine features of the distribution function profile. The advantage here is that all of the coefficients are linear. The results of our modeling of the electron VDFs shows that if you are able to resolve the spectral coefficients as a function of velocity and its moments by the spherical harmonic method, then you should be able to have a physical smooth representation of such velocity distributions that contains the main essential features that are supported by the observed measurements.

[32] The spherical harmonic method also provides a possible robust "compression" algorithm that can be easily implemented onboard any spacecraft to determine the moments and anisotropies of the velocity distribution function from particle detectors. In Cluster, for example, the data collected and used in the standard method of the moments of the velocity distribution function was 11,520 data measurements, but we have demonstrated that these moments can be accurately and effectively represented by $9 \times 30=$ 270 spectral coefficients. In general, returning moments computed onboard a spacecraft is problematic. Changes in instrument efficiencies and computation without taking into account the spacecraft potential makes such values suspect and often unusable. However, forming the moments from the spherical harmonic coefficients on the ground allows for both inclusion of the spacecraft potential and for changes in the instrument efficiency at the energy step level.

[33] An essential remark needs to be made at this point. We do not want to imply that the full data sample of the velocity distribution function are not required but just to suggest that in spacecraft missions where the details of the 


\section{Cluster Skymap of Electron Velocity Distribution Function \\ (SPH MODEL Lmax=5)}

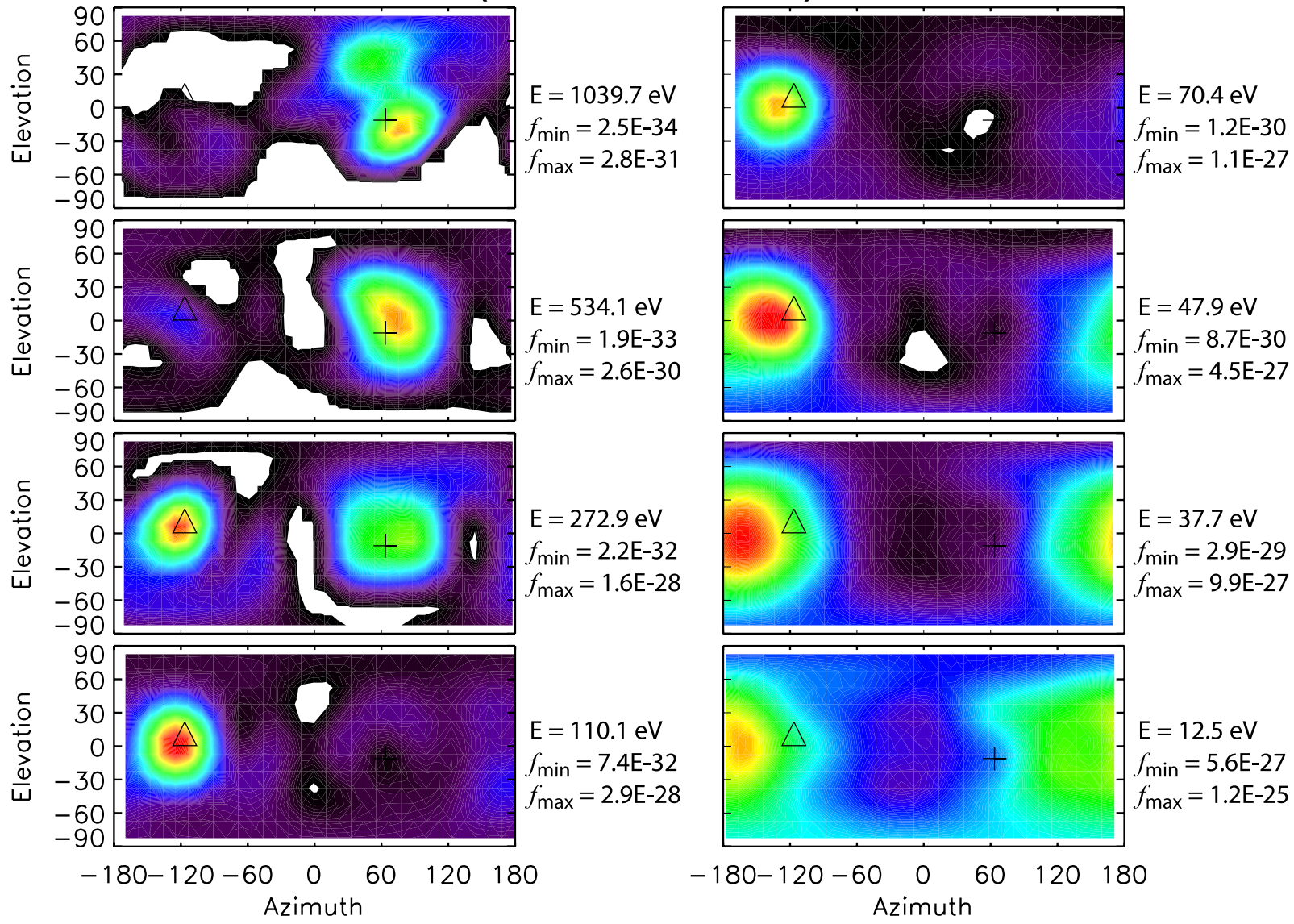

\section{6/078 Time 20:45:08}

Figure 8. Higher-resolution skymap multipanel plots of the spherical harmonic model for $l_{\max }=5$ (39 coefficients) of the velocity distribution function as a function of azimuth and elevation for different energy channels. Data are represented in the same frame as in Figure 6.

velocity distribution function are not that important and do not form part of the main science objectives, then the spherical harmonic approach can be an effective and robust approach for the determination of these fluids quantities. The method has also been tested using Cluster data in the magnetosheath and magnetosphere and the results are remarkably good and promising. It is also essential to test this method on regions where the fine details of the distribution function are more complex such as, for example, in other planetary magnetospheres and cometary environments. Our results are preliminary but they do show a promising approach to onboard computing, modeling, and full moment and anisotropies determination in space physics.

[34] Acknowledgments. We are grateful to M. L. Goldstein and A. Fazakerley for providing access to the electron velocity distribution data from the Cluster/PEACE instrument and for the various discussions and comments during the development of this work. A. F. Vinas would also like to thank Matt Holland for his assistance in the programming, Dean Pesnell for the many discussions, and Mark Adrian for his encouragement and support in the preparation of this work. This research was supported by NASA funding of the Cluster mission and PEACE experiment at the Goddard Space Flight Center.

[35] Amitava Bhattacharjee thanks Lev Zelenyi and another reviewer for their assistance in evaluating this paper.

\section{References}

Balogh, A., et al. (1997), The CLUSTER magnetic field investigation, Space Sci. Rev., 79, 65

Bayet, M. L., J. L. Delcroix, and J. F. Denisse (1954), Théorie cinétique des plasmas homogénex faiblement ionozés i, J. Phys. Radium, 15, 795.

Christensen-Dalsgaardhen, J. (2002), Helioseismology, Rev. Mod. Phys., 74, 1073.

Connerney, J. E. P. (1981), The magnetic field of Jupiter: A generalized inverse approach, J. Geophys. Res., 86, 7679.

Connerney, J. E. P., M. H. Acuña, and N. F. Ness (1991), The magnetic field of Neptune, J. Geophys. Res., 86, 19,023.

Feldman, W. C., J. R. Asbridge, S. J. Bame, M. D. Montgomery, and S. P. Gary (1975), Solar wind electrons, J. Geophys. Res., 80, 4181.

Grad, H. (1949), On the kinetic theory of rarefied gases, Commun. Pure Appl. Math., 2, 331.

Grant, F. C., and M. R. Feix (1967a), Fourier-Hermite solutions of the Vlasov equation in the linearized limit, Phys. Fluids, 10, 696.

Grant, F. C., and M. R. Feix (1967b), Transition between Landau and Van Kampen treatments of the Vlasov equation, Phys. Fluids, 10, 1356.

Gustafsson, G., et al. (1997), The electric field and wave experiment for the Cluster mission, Space Sci. Rev., 79, 137.

Jancel, R., and T. Kahan (1959a), Étude théorique de la distribution électronique dens un plasma Lorentzien hétérogene et anistrope, J. Phys. Radium, 1, 35.

Jancel, R., and T. Kahan (1959b), Méchanique statistique d'un plasma Lorentzien inhomogéne et anistrope: Étude de la distribution électronique, J. Phys. Radium, 1, 804

Jancel, R., and T. Kahan (1966), Electrodynamics of Plasmas, John Wiley, Hoboken, N. J. 
Johnson, T. W. (1960), Cartesian tensor scalar product and spherical harmonic expansions in Boltzmann's equation, Phys. Rev., 120, 1103.

Johnstone, A. D., et al. (1997), PEACE: A plasma electron and current experiment, Space Sci. Rev., 79, 351

Langel, R. A. (1987), Chapter 4, The Main Field, in Geomagnetism, vol. 1, edited by J. A. Jacobs, p. 249, Elsevier, New York.

Maksimovic, M. V., V. Pierrard, and P. Riley (1997), Ulysses electron distributions fitted with kappa functions, Geophys. Res. Lett., 24, 1151.

Maksimovic, M. V., et al. (2005), Radial evolution of the electron distribution functions in the fast solar wind between 0.3 and 1.5 AU, J. Geophys. Res., 110, A09104, doi:10.1029/2005JA011119.

Nieves-Chinchilla, T., and A. F. Viñas (2008), Solar wind electron distribution functions inside magnetic clouds, J. Geophys. Res., 113, A02105, doi:10.1029/2007JA012703.

Pilipp, W. G., H. Miggenrieder, M. D. Montgomery, K.-H. Mühlhaüser, H. Rosenbauer, and R. Schwenn (1987), Unusual electron distribution functions in the solar wind derived from the helios plasma experiment: Double-strahl distributions and distributions with an extremely anisotropic core, J. Geophys. Res., 92, 1093.

Press, W. H., S. A. Teukolsky, W. T. Vetterling, and B. P. Flannery (1999), Numerical Recipes in C: The Art of Scientific Computing, Cambridge Univ. Press, New York.
Ritzwoller, M. H., and E. M. Lavely (1991), A unified approach to the helioseismic forward and inverse problems of differential rotation, Astrophys. J., 369, 557.

Shakarofsky, I. P. (1963a), Cartesian tensor expansion of the Fokker-Planck equation, Can. J. Phys., 41, 1753.

Shakarofsky, I. P. (1963b), Inclusion of flow terms in the Cartesian tensor expansion of the Boltzmann equation, Can. J. Phys., 41, 1776.

Shakarofsky, I. P. (1963c), Calculation of the pressure tensor expansion in a fully ionized plasma, Can. J. Phys., 41, 1787.

Shkarofsky, I. P., T. W. Johnson, and M. P. Bachynski (1966), The Particle Kinetic of Plasmas, Addison-Wesley, Boston, Mass.

Skoug, R. M., W. C. Feldman, J. T. Gosling, D. J. McComas, and C. W. Smith (2000), Solar wind electron characteristics inside and outside coronal mass ejections, J. Geophys. Res., 105, 23,069.

C. Gurgiolo, Bitterroot Basic Research, 837 Westside Road, Hamilton, MT 59840, USA. (chris@gurgiolo.com)

A. F. Viñas, Heliophysics Science Division, Geospace Physics Laboratory, NASA Goddard Space Flight Center, Mail Code 673, Building 21/ Room 254, Greenbelt, MD 20771, USA. (adolfo.vinas@nasa.gov) 